\title{
Difficulties associated with removal of mesh eroding into vagina following abdominal sacrocolpopexy: a case report
}

\author{
H. Muppala $\cdot$ A. Bedoya-Ronga $\cdot$ M. Bhargava $\cdot$ \\ S. K. Najia
}

Received: 12 November 2006 / Accepted: 9 January 2007 / Published online: 30 January 2007

(C) Springer-Verlag 2007

\begin{abstract}
This case report describes the clinical case of a patient presenting complications during removal of mesh eroding through the lower one third of the posterior vaginal wall following abdominal sacrocolpopexy. Although excellent results have been reported with abdominal sacrocolpopexy for treatment of vaginal vault prolapse, minimizing complications and their correction remains a challenge. In this case, only $3 / 4$ of the mesh was removed vaginally and was complicated by small bowel perforation due to adhesions. The remaining mesh was removed by careful dissection from the sacral base as the risk of infection in the mesh left behind is increased. Successful management eventually requires complete removal of the mesh at laparotomy. The surgery should be performed by experienced pelvic surgeons able to resolve intraoperative complications. Although serious complications are rare, patients should nonetheless be counselled about the risk of massive bleeding, bowel perforation, infection and rectovaginal fistula formation.
\end{abstract}

Keywords Abdominal sacrocolpopexy $\cdot$ Mesh erosion

\section{Case report}

A 51-year-old patient underwent two diagnostic laparoscopies in 1981 for chronic pelvic pain and subsequently had laparoscopic adhesiolysis in 1982. In 1989, the patient underwent total abdominal hysterectomy for menorrhagia

H. Muppala $(\bowtie) \cdot$ A. Bedoya-Ronga $\cdot$ M. Bhargava $\cdot$ S. K. Najia Women's Health Directorate, Royal Blackburn Hospital,

Haslingden Road, Blackburn,

BB2 3HH Lancashire, UK

e-mail: drmuppala@dochdoc.com and in April 2004, anterior and posterior colpoperineorrhaphy. In May 2005 she underwent abdominal sacrocolpopexy for vault prolapse and, in view of extensive pelvic adhesions and matting of both the fallopian tubes and ovaries, adhesiolysis with bilateral salphingo-oophorectomy was performed. The mesh was anchored in the rectovaginal space with PDS to act both as vault suspension and in preventing rectocele. It was then fixed to the sacrum at the sacral promontory to the anterior sacral ligament with nylon. The mesh was placed in the retroperitoneal space and re-peritonealised after securing at both the ends. $6 \times 6$ inches of folded Prolene mesh is used for vault suspension. This mesh is a non-absorbable inert, sterile, porous surgical mesh knitted from monofilament fibers of polypropylene polymer (Surgipro mesh, Surgipro, USA) and measures approximately $0.57 \mathrm{~mm}$ in thickness. Medical history of the patient revealed NIDDM on diet control, high blood pressure and a smoker of 20 cigarettes a day. Fifteen months later the patient presented with postmenopausal bleeding and on examination mesh eroding through the lower $1 / 3$ of the posterior vaginal wall was noted. Mesh was gently released from fibrous tissue until 3/4 of the mesh was dissected, cut and removed. Small bowel perforation was noticed by the presence of bile, where it was adherent to the vaginal vault. A defect in the vaginal wall was closed continuously taking the support of the fibrous tissue on the pelvic side walls. On midline laparotomy small bowels were all densely adhered together and into the pelvic cavity. The vaginal vault itself appeared to be well supported by the formation of fibrous tissue. By gentle dissection small bowels were released and the perforated area identified. Small bowel resection and end to end single layer anastomosis was performed. The remaining mesh and suture were removed at laparotomy by careful dissection of the fibrous tissue from the sacral attachment. 
Small bleeding points were controlled from the sacral base by pressure and placement of Surgicel mesh (oxidized cellulose) (Ethicon, Inc., USA). The patient received antibiotic prophylaxis with cefuroxime and metronidazole postoperatively for 5 days and was discharged home uneventfully on day five.

\section{Discussion}

The incidence of post hysterectomy vaginal vault prolapse ranges from $0.2-1 \%$ [1]. The sacrocolpopexy, a widely accepted and excellent procedure to surgically treat vaginal vault prolapse, has success rates ranging from $84-99 \%$ [2], with the ability to restore the vaginal axis without compromising vaginal depth and also correct rectocele. A synthetic graft is often used to support the vaginal apex, but has the potential to become infected or erode, requiring its removal or revision. The overall rate of erosion per procedure is $2.7 \%$ [3]. Although the procedure boasts excellent success rates, minimizing complications remains a challenge. The sacrocolpopexy interposes material from the vaginal cuff to the longitudinal ligament of the spine. This introduces foreign body that is capable of affecting tissues in proximity, either by direct contact or by harboring infection that is difficult to eradicate without complete graft removal. This is because permeation of antibiotics into the mesh is less likely due its porous nature and fibrous tissue formation. But its management is not without complications. Partial removal may result in sinus tract formation, recurrence of mesh erosion [3] or infection of the remaining mesh and its risk is further enhanced with bowel perforation. Careful and meticulous dissection of the fibrous tissue formed by the sides of mesh is required at the sacral end, as proximity to presacral veins increases risk of bleeding which at times can be life-threatening [1]. Successful management eventually requires complete removal of mesh at laparotomy. The operative technique is simple but the surgery should be performed by experienced pelvic surgeons able to resolve intraoperative complications. Although rare, patients should be counseled about serious complications such as massive bleeding, bowel perforation, infection, sinus tract and rectovaginal fistula formation [4, 5]. Mesh erosion is an unfortunate complication of abdominal sacral colpopexy and the search for the best graft material continues.

\section{References}

1. Kohli N, Walsh PM, Roat TW, Karram MM (1998) Mesh erosion after abdominal sacrocolpopexy. Obstet Gynecol 92:999-1004

2. Bensinger G, Lind L, Lesser M, Guess M, Winkler HA (2005) Abdominal sacral suspensions: analysis of complications using permanent mesh. Am J Obstet Gynaecology 193:2094-2098

3. Begley JS et al (2005) Incidence and management of abdominal sacrocolpopexy mesh erosions. Am J Obstet Gynaecology 192:1956-1962

4. Hart SR, Weiser EB (2004) Abdominal sacral colpopexy mesh erosion resulting in a sinus tract formation and sacral abscess. Obstet Gynecol 103:1037-1040

5. Hopkins MP, Rooney C (2004) Entero mesh vaginal fistula secondary to abdominal sacral colpopexy. Obstet Gynecol 103:1035-1036 